\title{
67. PARTICLE RESONANCE IN A SPIRAL FIELD
}

\author{
B. BAR BANIS
}

Dept. of Astronomy, University of Patras, Greece

\begin{abstract}
Plane galactic orbits near the particle resonance of a spiral field have been calculated numerically. Besides the ring type orbits, i.e. orbits filling a ring around the galactic center, there are orbits librating near the potential maxima at the co-rotation distance (Lagrangian points). This effect tends to disrupt the spiral arms at the co-rotation distance.
\end{abstract}

A large number of particle plane orbits has been calculated in a typical logarithmic spiral potential superimposed on an axisymmetric potential which represents the Contopoulos-Strömgren (1965) model of the force field on the galactic plane. The spiral force field used is about $7 \%$ of the axisymmetric field (Barbanis and Woltjer, 1967; Barbanis, 1968a, b).

Expressing the distance to the galactic center $\varpi$ in $\mathrm{kpc}$ and the unit of time in $10^{7} \mathrm{yr}$ the axisymmetric potential is

$$
\begin{aligned}
& \phi_{0}=1.0459\left(-7.334 \varpi^{-1}-0.15818 \varpi+0.1721015 \varpi^{2}-\right. \\
&-\left.0.0134207 \varpi^{3}+0.000323505 \varpi^{4}\right) .
\end{aligned}
$$

The adopted logarithmic spiral potential, consisting of two arms and rotating as a rigid body with an angular velocity $\Omega_{\mathrm{s}}$, is

$$
\varepsilon \phi_{\mathrm{s}}=\varepsilon \varpi^{1 / 2}(16-\varpi) \cos \left\{2 \varphi-2 \Omega_{\mathrm{s}} t+20 \ln ((\varpi+4) / 8)\right\},
$$

where $\varpi, \varphi$ are polar coordinates with respect to an inertial frame and $\varepsilon=0.001$.

The angular velocity of the spiral pattern used is $\Omega_{\mathrm{s}}=25 \mathrm{~km} \mathrm{~s}^{-1} \mathrm{kpc}^{-1}$ and it is equal to the angular velocity $\Omega$ of the axisymmetric model at $10 \mathrm{kpc}$. This value is larger than Lin's value (Lin et al., 1969) by a factor of 2 , but close to the values given by Kalnajs during this Symposium (this volume, p. 318) and Marochnik and Suchkov (1969). However, the main purpose of this paper is to investigate the particle resonance phenomena wherever they appear.

The equations of motion of a mass point with coordinates $(x, y)$ in a reference frame rotating with angular velocity $\Omega_{\mathrm{s}}$ are

$$
\begin{aligned}
& \frac{\mathrm{d}^{2} x}{\mathrm{~d} t^{2}}=\Omega_{\mathrm{s}}\left(2 \frac{\mathrm{d} y}{\mathrm{~d} t}+\Omega_{\mathrm{s}} x\right)-\frac{\partial \phi}{\partial x}, \\
& \frac{\mathrm{d}^{2} y}{\mathrm{~d} t^{2}}=-\Omega_{\mathrm{s}}\left(2 \frac{\mathrm{d} x}{\mathrm{~d} t}-\Omega_{\mathrm{s}} y\right)-\frac{\partial \phi}{\partial y},
\end{aligned}
$$

where $\phi=\phi_{0}+\varepsilon \phi_{\mathrm{s}}$; the argument of the cosine in $\phi_{\mathrm{s}}$ is

$$
2 \theta=2 \tan ^{-1}(y / x)+20\left\{\ln [(\varpi+4) / 8]-\ln \left[\left(\varpi_{0}+4\right) / 8\right]\right\},
$$


where $\varpi, \theta$ are polar coordinates in the rotating frame with origin the galactic center; the $x$-axis intersects the spiral arm at a distance $\varpi_{0}=10 \mathrm{kpc}$.

The Jacobi integral is

$$
H=\frac{1}{2}\left(X^{2}+Y^{2}-\Omega_{\mathrm{s}}^{2} \varpi^{2}\right)+\phi,
$$

where $X=\mathrm{d} x / \mathrm{d} t, Y=\mathrm{d} y / \mathrm{d} t$, and $\varpi^{2}=x^{2}+y^{2}$.

If $H$ is smaller than $1.67\left(\mathrm{kpc} / 10^{7} \mathrm{yr}\right)^{2}$ the curve of zero velocity consists of two near circles (dotted curves) around the galactic center G.C. (Figure 1). The velocity is real inside the inner curve or outside the outer curve. The co-rotation point CR on the $x$-axis lies inside the ring, where the motion is not permitted. The width of this ring gradually decreases as the value of $H$ increases and tends to the value 1.67.

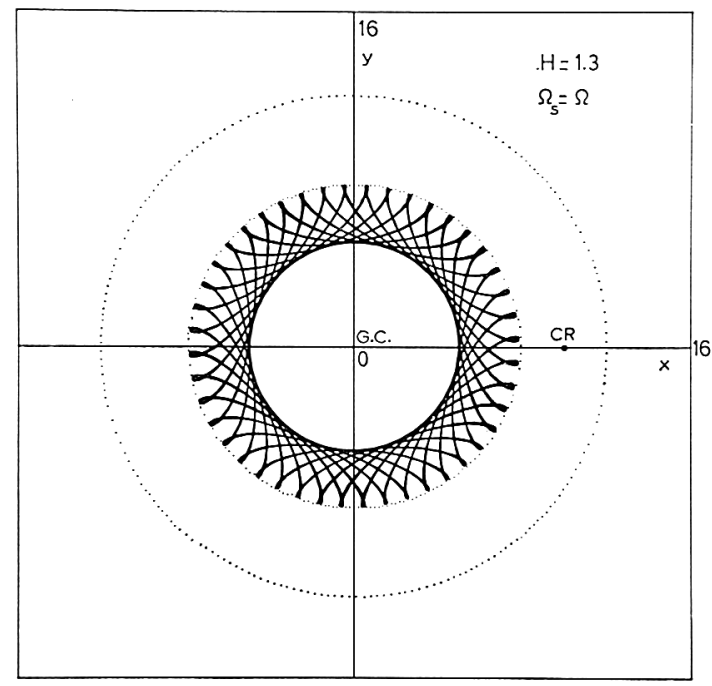

Fig. 1 A ring type orbit for $H=1.3, \Omega_{\mathrm{s}}=\Omega=25 \mathrm{~km} \mathrm{~s}^{-1} \mathrm{kpc}^{-1}$, and initial conditions: $x_{0}=5 \mathrm{kpc}$, $y_{0}=0, X_{0}=0$. G.C. is the galactic center and CR the co-rotation point on the $x$-axis. The curve of zero velocity (dotted curves) consists of two near circles; the motion is not permitted inside the ring.

Orbits 'near' a circular orbit, starting inside the inner curve, fill rings around the origin with their external boundary inside the curve of zero velocity. The corresponding invariant curves on the plane $x X$, for $x>0$, are inside a boundary (Figure 2). Each invariant curve of Figure 2 is marked by two numbers giving the initial distance and radial velocity (the initial point is always on the $x$-axis). Orbits starting near the periodic orbit have closed invariant curves, but the invariant curves of orbits further away are open and terminate on the boundary. The terminal points correspond to those loops of an orbit (Figure 1) which are tangent to the $x$-axis.

For $H$ larger than 1.67 the zero velocity curve disappears and the motion is now permitted everywhere on the $x y$-plane. However, some of the orbits fill again a ring around the galactic center. This leads to the conclusion that besides the energy integral there is another integral which restricts the motion in a finite area. 
The most important effect near the co-rotation point is a new type of orbits, which appear for $H>1.67$ and a certain range of initial conditions. These orbits librate up and down with respect to the $x$-axis. Figure 3 gives two such librating orbits for $H=1.7$ and initial conditions: $x_{0}= \pm 8.5 \mathrm{kpc} y_{0}=X_{0}=0$. The co-rotation points, on

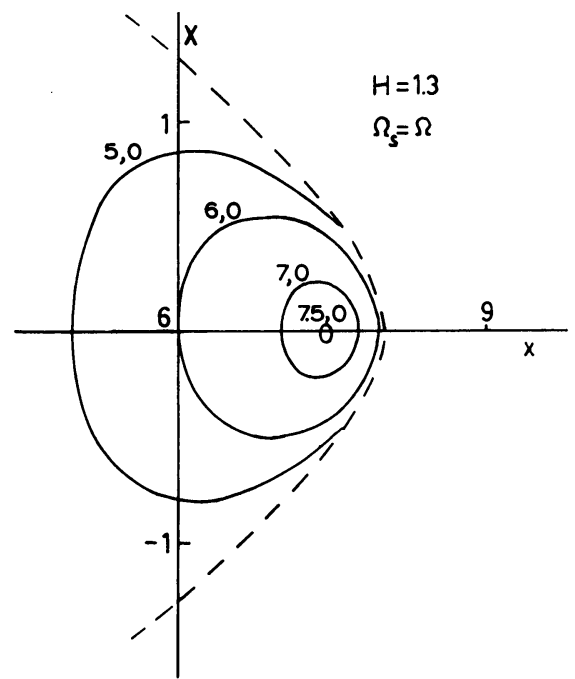

Fig. 2. Invariant curves of various orbits for $H=1.3$. The dashed line gives the boundary of the invariant curves on the $x X$-plane for orbits starting inside the inner zero velocity curve. The two numbers on each invariant curve give the initial position in $\mathrm{kpc}$ and the radial velocity in $\mathrm{km} \mathrm{s}^{-1}$ of the moving point.

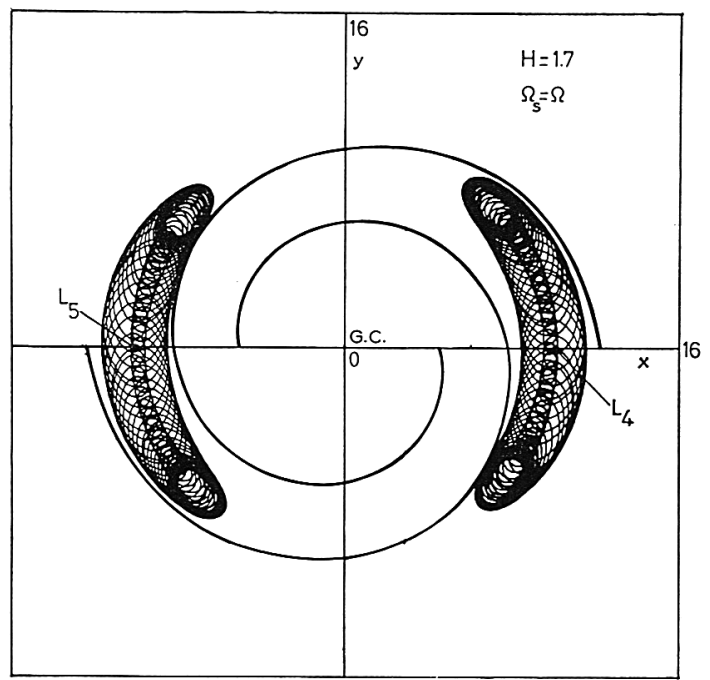

Fig. 3. Two librating orbits for $H=1.7$ and $x_{0}= \pm 8.5 \mathrm{kpc}, y_{0}=X_{0}=0$. The two spiral arms give the density maxima. $L_{4}$ and $L_{5}$ are the co-rotation points on the $x$-axis, where the potential is maximum; these points correspond to the Lagrangian points $L_{4}, L_{5}$ of the restricted three-body problem. 
the $x$-axis, lie near the middle between the extreme intersections of the $x$-axis by each orbit. At these points the spiral potential is maximum. These points correspond to the Lagrangian points $L_{4}, L_{5}$ of the restricted three-body problem. The librating orbits correspond to the Trojan orbits near $L_{4}$ and $L_{5}$.

Such librating orbits appear if $8.3<\left|x_{0}\right|<9.8 \mathrm{kpc}$. One orbit near each Lagrangian point $L_{4}, L_{5}$ is periodic. Orbits near it fill an elongated ring surrounding the periodic orbit (Figure 4). For $\left|x_{0}\right| \geqslant 9.8 \mathrm{kpc}$ the orbits fill again rings around the galactic center.

For $H=1.7$ the boundaries of the invariant curves (Figure 5; dashed lines) do not intersect the $x$-axis. For each orbit, filling a ring around the galactic center, correspond two open invariant curves, one with positive $x$ and the other with negative $x$. On the other hand, the invariant curves of librating orbits are closed curves or two open curves situated on one side of the $X$-axis.

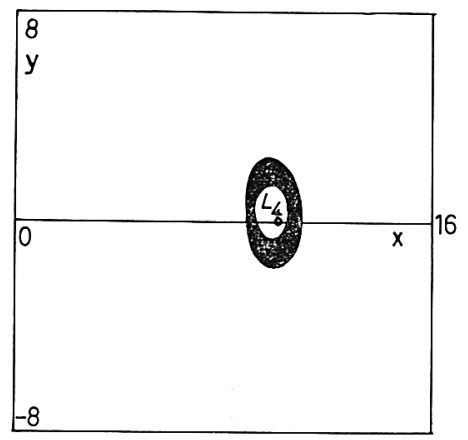

Fig. 4. An orbit for $H=1.7$ and $x_{0}=9.1 \mathrm{kpc}, y_{0}=X_{0}=0$. Such an orbit fills an elongated ring around $L_{4}$.

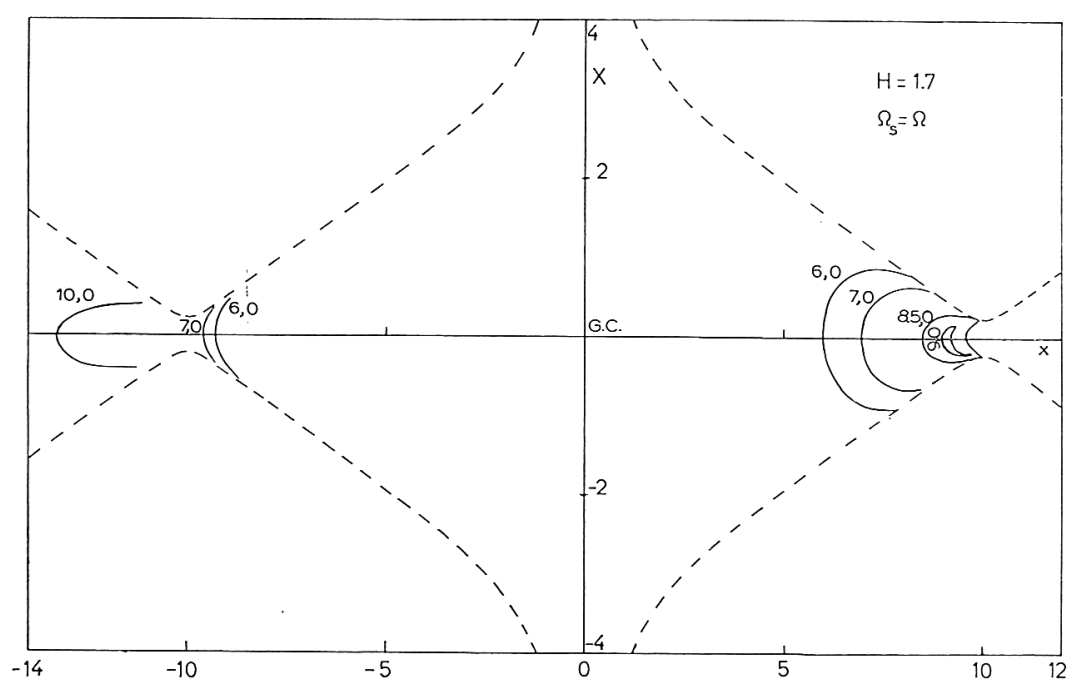

Fig. 5. Invariant curves of various orbits calculated for $H=1.7$. Ring type orbits produce two open invariant curves one at the positive and the other at the negative part of the $x$-axis. Invariant curves of librating orbits are closed, or two open curves situated on the same side of the $X$-axis. 
Similar results were found for $H=2$. Therefore librating orbits appear for a substantial range of initial conditions near the co-rotation distance. We conclude that an appreciable amount of matter in this neighbourhood is concentrated near the Lagrangian points $L_{4}, L_{5}$. Matter goes away from the minima of potential (the outermost points of intersection of the spiral arms of Figure 3 by the $y$-axis) and forms two density maxima near the Lagrangian points; this phenomenon is well known in celestial mechanics.

The consequence for the Galaxy is a disruption of the observed spiral arms at the co-rotation distance. Self-consistent spiral waves can exist inside or outside the corotation point, but near this point non-linear effects produce their breaking. This effect may be connected with the observed broken form of the spiral arms in the outer parts of many galaxies.

\section{References}

Barbanis, B.: 1968a, Astrophys. J. 153, 71.

Barbanis, B.: 1968b, Astron. J. 73, 784.

Barbanis, B. and Woltjer, L.: 1967, Astrophys. J. 150, 461.

Contopoulos, G. and Strömgren, B.: 1965, Tables of Plane Galactic Orbits, Inst. Space Studies, New York.

Lin, C. C., Yuan, C., and Shu, F.: 1969, Astrophys. J. 155, 721.

Marochnik, L. S. and Suchkov, A. A.: 1969, Astrophys. Space Sci. 4, 317. 